\title{
Vanishing torque from radiation pressure (Research Note)
}

\author{
D. Nesvorný ${ }^{1,2}$ and D. Vokrouhlický1,3 \\ 1 Department of Space Studies, Southwest Research Institute, 1050 Walnut St., Suite 300, Boulder, Colorado 80302, USA \\ e-mail: davidn@boulder.swri.edu \\ 2 Observatório Nacional, R. Gal. José Cristino 77, 20921-400 Rio de Janeiro, Brazil \\ 3 Institute of Astronomy, Charles University, V Holešovičkách 2, 18000, Prague 8, Czech Republic
}

Received 31 July 2007 / Accepted 5 November 2007

\section{ABSTRACT}

\begin{abstract}
Context. Pioneering works on the effect of solar irradiation on the rotation of small objects in interplanetary space raised the possibility that the radiation pressure from solar photon absorption could modify the objects' spin states. Later numerical studies found that the torque from radiation pressure, when averaged over spin and orbital periods, vanished for all studied asteroid shape models. Aims. We demonstrate that the average torque from radiation pressure vanishes for any surface shape deformation.

Methods. We have calculated the torque analytically. The main assumption of our calculation is that the object rotates around its principal axis of inertia.

Results. We show that the average torque vanishes for any surface shape deformation because the individual radiation-pressure contributions from configurations separated by $\pi$ in orbital longitude cancel each other out.

Conclusions. Unlike the thermal radiation and reflection of solar photons from surface, which can produce important effects over planetary timescales, the radiation pressure cannot change asteroid rotation.
\end{abstract}

Key words. minor planets, asteroids

\section{Introduction}

The surface of an irregularly-shaped small object in the interplanetary space is irradiated by sunlight. The torque produced by absorbed photons is

$\boldsymbol{\tau}=-\frac{F}{h^{2} c} \int_{S} \mathrm{~d} S\left(\boldsymbol{r} \times \boldsymbol{n}_{0}\right)\left(\boldsymbol{n} \cdot \boldsymbol{n}_{0}\right)$,

where the integral is taken over the illuminated part of the surface. Here, $c$ is the speed of light, $F=1378 \mathrm{~W} \mathrm{~m}^{-2}$ the solar flux at distance $1 \mathrm{AU}$ from the Sun, and $h$ the heliocentric distance of the object in AU. Vectors $\boldsymbol{n}$ and $\boldsymbol{n}_{0}$ are unit vectors pointing from surface element $\mathrm{d} S$ in normal and toward-the-Sun directions, respectively. Vector $\boldsymbol{r}$ connects the center of mass of the object to $\mathrm{d} S$.

In addition to (1), two other torque components arise from the interaction of the surface with solar radiation. These are produced by (i) reflected solar photons and (ii) thermal photons departing from the surface heated by sunlight. The total torque, called the YORP torque (after researchers Yarkovsky, O'Keefe, Radzievskii, and Paddack; Rubincam 2000), can be obtained by adding torque components together. The YORP effect has important applications in asteroid spin dynamics (e.g., Vokrouhlický et al. 2003, 2007; Bottke et al. 2006).

We limit the analysis in this paper to the Keplerian orbital motion of a small body around the Sun and its rotation around the principal axis of inertia. The latter assumption is justified for $\mathrm{km}$-sized and larger asteroids because any excess in the rotational energy can be efficiently dissipated in their interior (e.g., Sharma et al. 2005).
To study secular spin dynamics, it is convenient to average $\tau$ over the spin and orbit periods of the small object (Rubincam 2000). The mean torque, $\bar{\tau}$, is defined as

$\bar{\tau}=\frac{1}{(2 \pi)^{2}} \int_{0}^{2 \pi} \int_{0}^{2 \pi} \tau \mathrm{d} \phi_{0} \mathrm{~d} \lambda$,

where $\phi_{0}$ is the spin phase angle (see Sect. 2) and $\lambda$ the mean longitude of the small body presumed here to be orbiting around the Sun on a fixed orbit.

Early studies of the YORP effect discussed the possibility whether (2) can produce changes in the spin state of a small object. Modern numerical works have found that (2) vanished in every studied case, including asteroids with known shape models and artificial objects (Rubincam 2000; Vokrouhlický \& Čapek 2002). Here we calculate $\bar{\tau}$ analytically. Our results imply that $\bar{\tau}=0$ for any shape deformation. Accordingly, the radiation pressure does not secularly affect principal axis rotation.

This note is organized as follows. In Sect. 2, we introduce useful reference frames. In Sect. 3, we show that the instantaneous torque from the radiation pressure only depends on the object's silhouette as seen from the Sun. Finally, in Sect. 4, we demonstrate that the torque vanishes when it is averaged over the orbital period.

\section{Reference frames}

We use several reference frames all with the origin at the center of mass of the small object (Nesvorný \& Vokrouhlický 2007). Figure 1 illustrates these frame systems. The body frame, $O x^{\prime \prime} y^{\prime \prime} z^{\prime \prime}$, has the $z^{\prime \prime}$ axis fixed along the object's spin axis (assumed here to coincide with the principal inertia axis) and the 


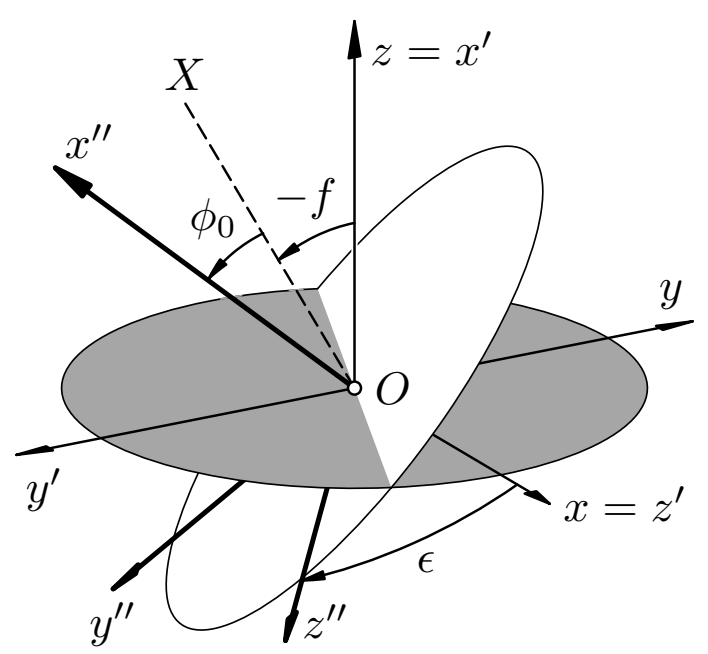

Fig. 1. The illustration of various reference frames used in this study. $O x y z, O x^{\prime} y^{\prime} z^{\prime}$, and $O x^{\prime \prime} y^{\prime \prime} z^{\prime \prime}$ denote the solar, rotating orbital, and body frames, respectively. See Sect. 2 for the definition of these reference systems. The body frame has been obtained from the rotating orbital frame via three rotations indicated here (Eq. (3)).

$x^{\prime \prime}$ axis along its shortest axis of inertia. The rotating orbital frame, $O x^{\prime} y^{\prime} z^{\prime}$, has the $z^{\prime}$ axis pointing toward the normal of the orbital plane and the $x^{\prime}$ axis pointing toward the Sun. The transformation of a vector from the rotating orbital frame to the body frame is given by

$V^{\prime \prime}=R_{3}\left(\alpha_{3}\right) R_{1}\left(\alpha_{2}\right) R_{3}\left(\alpha_{1}\right) V^{\prime}$

with Euler angles $\alpha_{1}=-f, \alpha_{2}=\epsilon$ and $\alpha_{3}=\phi_{0}$, where $\phi_{0}=\omega t$ denotes the phase angle of the body's rotation with respect to the inertial frame, $\omega$ is the angular frequency of rotation, $\epsilon$ the obliquity, and $f$ the true orbital longitude of the Sun. Symbols $R_{1}$ and $R_{3}$ in Eq. (3) are the usual rotation matrices that represent the rotation of the reference system around the (generic) $x$ and $z$ axes, respectively.

Our third reference system is the frame with the $z$ axis pointing toward the Sun and the $x$ axis pointing toward the normal of the orbital frame. We call this reference system the solar frame. This frame rotates in an inertial system with the angular speed given by the orbital motion of the small body around the Sun. The transformation of any vector $\boldsymbol{V}$ from the solar frame to the rotating orbital frame is

$\boldsymbol{V}^{\prime}=R_{3}\left(\alpha_{3}\right) R_{1}\left(\alpha_{2}\right) R_{3}\left(\alpha_{1}\right) \boldsymbol{V}$

with Euler angles $\alpha_{1}=\alpha_{2}=\alpha_{3}=\pi / 2$. The colatitude and longitude in the solar frame is denoted by $\theta$ and $\phi$.

\section{Instantaneous torque in the solar frame}

We calculate Eq. (1) in the solar frame where $\boldsymbol{n}_{0}=(0,0,1)^{\mathrm{T}}$ with index $\mathrm{T}$ denoting the transposed vector. Because $\boldsymbol{n} \mathrm{d} S=$ $N \mathrm{~d} \theta \mathrm{d} \phi=N(\mathrm{~d} \Omega / \sin \theta)$, Eq. (1) can be written as

$\boldsymbol{\tau}=-\alpha \int_{\Omega} \frac{\mathrm{d} \Omega}{\sin \theta}\left(\boldsymbol{r} \times \boldsymbol{n}_{0}\right)\left(\boldsymbol{N} \cdot \boldsymbol{n}_{0}\right)$,

where $\alpha=F /\left(h^{2} c\right), \mathrm{d} \Omega=\sin \theta \mathrm{d} \theta \mathrm{d} \phi, \boldsymbol{n}=\boldsymbol{N} /|\boldsymbol{N}|, \boldsymbol{N}=\boldsymbol{t}_{\theta} \times \boldsymbol{t}_{\phi}$, and where $\boldsymbol{t}_{\theta}=\partial \boldsymbol{r} / \partial \theta$ and $\boldsymbol{t}_{\phi}=\partial \boldsymbol{r} / \partial \phi$ are tangential vectors. The integration over solid angle $\Omega$ goes over the illuminated part of the surface. Assuming $r=r(\phi, \theta)$, which is appropriate for all shapes except the ones for which radial ray $(\theta, \phi)$ can intersect the surface in more than one point, it can be easily shown that $\boldsymbol{t}_{\theta}=r_{\theta} \boldsymbol{u}_{r}+r \boldsymbol{u}_{\theta}$ and $\boldsymbol{t}_{\phi}=r_{\phi} \boldsymbol{u}_{r}+r \sin \theta \boldsymbol{u}_{\phi}$, where $r_{\theta}=\partial r / \partial \theta$ and $r_{\phi}=\partial r / \partial \phi$. The vectors $\boldsymbol{u}_{r}, \boldsymbol{u}_{\theta}$, and $\boldsymbol{u}_{\phi}$ used above were defined as

$$
\boldsymbol{u}_{r}=\left(\begin{array}{c}
\sin \theta \cos \phi \\
\sin \theta \sin \phi \\
\cos \theta
\end{array}\right), \boldsymbol{u}_{\theta}=\left(\begin{array}{c}
\cos \theta \cos \phi \\
\cos \theta \sin \phi \\
-\sin \theta
\end{array}\right), \boldsymbol{u}_{\phi}=\left(\begin{array}{c}
-\sin \phi \\
\cos \phi \\
0
\end{array}\right) \text {. }
$$

They form an orthonormal triad (i.e., $\boldsymbol{u}_{r}=\boldsymbol{u}_{\theta} \times \boldsymbol{u}_{\phi}$, etc.). Using these vectors the expression for $N$ reads as

$\boldsymbol{N}=r\left[r \sin \theta \boldsymbol{u}_{r}-r_{\theta} \sin \theta \boldsymbol{u}_{\theta}-r_{\phi} \boldsymbol{u}_{\phi}\right]$

therefore,

$\boldsymbol{N} \cdot \boldsymbol{n}_{0}=N_{z}=r \sin \theta\left(r \cos \theta+\frac{\partial r}{\partial \theta} \sin \theta\right)$,

where $N_{z}$ is the z-component of $\boldsymbol{N}$ in the solar frame. We also have in the solar frame that

$\boldsymbol{r} \times \boldsymbol{n}_{0}=-r \sin \theta \boldsymbol{u}_{\phi}$.

Now, substituting the above expressions into (5), we find that

$$
\begin{aligned}
\boldsymbol{\tau} & =\alpha \int_{\Omega} \frac{\mathrm{d} \Omega}{\sin \theta} \boldsymbol{u}_{\phi} r^{2} \sin ^{2} \theta\left(r \cos \theta+\frac{\partial r}{\partial \theta} \sin \theta\right) \\
& =\frac{\alpha}{3} \int_{\Omega} \frac{\mathrm{d} \Omega}{\sin \theta} \boldsymbol{u}_{\phi} \frac{\partial}{\partial \theta}\left(r^{3} \sin ^{3} \theta\right) \\
& =\frac{\alpha}{3} \int_{0}^{2 \pi} \mathrm{d} \phi \boldsymbol{u}_{\phi} \int \mathrm{d} \theta \frac{\partial}{\partial \theta}\left(r^{3} \sin ^{3} \theta\right) .
\end{aligned}
$$

The above integral over $\theta$ needs to be taken over the illuminated part of the surface. The illuminated part of the surface of an ideally spherical object is its whole hemisphere facing the Sun. In such an idealized case, the integral over $\theta$ would go from 0 to $\pi / 2$. For a general surface deformation for which shadowing of surface elements can occur, the integral over $\theta$ can be written as

$\int \mathrm{d} \theta \frac{\partial}{\partial \theta}\left(r^{3} \sin ^{3} \theta\right)=\sum_{j}\left[r^{3} \sin ^{3} \theta\right]_{\theta_{1, j}}^{\theta_{2, j}}$,

where $\theta_{1, j}(\phi)$, and $\theta_{2, j}(\phi)$ denote the latitude limits of illuminated segments stretching along the body's meridian (with constant $\phi$ ). Given the illumination geometry in the solar frame, we note that $r\left(\theta_{2, j}, \phi\right) \sin \theta_{2, j}=r\left(\theta_{1, j+1}, \phi\right) \sin \theta_{1, j+1}$ for all $j$. Therefore, although in general discontinuous in $\theta$, Eq. (11) is continuous in $r \sin \theta=\sqrt{x^{2}+y^{2}}$, where $x$ and $y$ are the Cartesian coordinates in the solar frame. Equation (10) can be reduced to

$\boldsymbol{\tau}=-\frac{\alpha}{3} \int_{0}^{2 \pi} \mathrm{d} \phi \rho(\phi)^{3} \boldsymbol{u}_{\phi}$

where $\rho(\phi)=r\left(\theta_{\max }, \phi\right) \sin \theta_{\max }$ with $\theta_{\max }=\theta_{\max }(\phi)$ denoting the value of $\theta$ at the terminator.

The meaning of (12) is clear: the instantaneous torque from the radiation pressure only depends on the silhouette that the irregular object presents to the Sun (Rubincam 2000). It is straightforward to show that the same result also holds for a more general case of surface deformation where radial ray $(\theta, \phi)$ is allowed to intersect the surface at more than one point.

Until now we have assumed that the object is illuminated with $\boldsymbol{n}_{0}^{(+)}=\boldsymbol{n}_{0}=(0,0,1)^{\mathrm{T}}$ in the solar frame. If, instead, the object is illuminated from the opposite direction, i.e. with 
$\boldsymbol{n}_{0}^{(-)}=-\boldsymbol{n}_{0}=(0,0,-1)^{\mathrm{T}}$, the silhouette of the object and therefore also the magnitude of the torque would be the same. It is easy to show, moreover, that the instantaneous torque vector produced with $\boldsymbol{n}_{0}^{(-)}$goes in the opposite direction to the one obtained with $\boldsymbol{n}_{0}^{(+)}$; i.e., $\boldsymbol{\tau}\left(\boldsymbol{n}_{0}^{(-)}\right)=-\boldsymbol{\tau}\left(\boldsymbol{n}_{0}^{(+)}\right)$. This last statement stems from the following. With $\boldsymbol{n}_{0}^{(-)}$, Eq. (10) remains the same except for the integration domain that now spans over the opposite hemisphere of the object than before; the integration path in $\theta$ must be changed accordingly. We obtain

$\boldsymbol{\tau}\left(\boldsymbol{n}_{0}^{(-)}\right)=\frac{\alpha}{3} \int_{0}^{2 \pi} \mathrm{d} \phi \rho(\phi)^{3} \boldsymbol{u}_{\phi}$,

as claimed above.

\section{Vanishing mean torque}

To determine average torque (2), we need to transform the torque vector from the solar frame to the body frame and average it over $\lambda$ and $\phi_{0}$. We note that the integration over $\lambda$ can be simplified by introducing true longitude $f$ as the new integration variable:

$$
\int \mathrm{d} \lambda \tau=\frac{1}{\sqrt{1-e^{2}}} \int \mathrm{d} f\left(\frac{h}{a}\right)^{2} \tau .
$$

The dependence on $h$ then conveniently cancels out from (14) because $\tau \propto h^{-2}$ according to (1). We therefore find that the stronger torques produced by sunlight at the perihelion are compensated for by the shorter time interval that an asteroid spends near perihelion.

In the body frame, the object presents different silhouettes to the Sun depending on the Sun's location. With the help of transformation rules (3) and (4), we can transform the vector $\boldsymbol{n}_{0}$ from the solar frame, where $\boldsymbol{n}_{0}=(0,0,1)^{\mathrm{T}}$, to the body frame, where

$\boldsymbol{n}_{0}=\left(\begin{array}{c}\cos \phi_{0} \cos f+\sin \phi_{0} \sin f \cos \epsilon \\ -\sin \phi_{0} \cos f+\cos \phi_{0} \sin f \cos \epsilon \\ -\sin f \sin \epsilon\end{array}\right)$.

In the course of its spin and orbital motion, the object shows different sides of its figure to the Sun. Yet, it is clear that its silhouettes as seen from the Sun are the same for $\boldsymbol{n}_{0}$ and $-\boldsymbol{n}_{0}$.
This reversal of the Sun's direction in the body frame is achieved when $f$ is substituted by $f+\pi$ in (15). According to the results in the previous section, the instantaneous torque vectors for $\left(f, \phi_{0}\right)$ and $\left(f+\pi, \phi_{0}\right)$ then cancel when added together. Therefore, the integral over $\lambda$ in (2) vanishes. We conclude that

$\bar{\tau}=0$.

\section{Conclusions}

Our result implies that the average torque from the radiation pressure vanishes for any surface shape deformation. This represents an important generalization from the previous analytical results (e.g., Breiter et al. 2007) and shows that the radiation pressure effects cannot change asteroid rotation.

According to (12), the radiation pressure can only apply a tiny and variable instantaneous torque to the rotating body. The physical importance of this effect is negligible in the present studies of asteroidal dynamics. Still, in principle, the varying radiation pressure torques could cause small periodical changes in an asteroid's spin state. It is even possible, in the absence of efficient dissipation of the excess spin energy, that these variable torques could produce slight deviations of the spin vector from the principal axis. Our assumption of principal axis rotation must be relaxed to make theoretical investigations into such matters possible.

Acknowledgements. The funding for this work was provided by NASA's Planetary Geology and Geophysics program, and by CNPq. The work of D.V. was supported by the Grant Agency of the Czech Republic (grant 205/05/2737). We thank Miroslav Brož for Fig. 1.

\section{References}

Bottke, W. F., Vokrouhlický, D., Rubincam, D. P., \& Nesvorný, D. 2006, ARA\&A, 34, 157

Breiter, S., Michalska, H., Vokrouhlický, D., \& Borczyk, W. 2007, A\&A, 471, 345

Nesvorný, D., \& Vokrouhlický, D. 2007, AJ, 134, 1750

Rubincam, D. P. 2000, Icarus, 148, 2

Sharma, I., Burns, J. A., \& Hui, C.-Y. 2005, MNRAS, 359, 79

Vokrouhlický, D., \& Čapek, D. 2002, Icarus, 159, 449

Vokrouhlický, D., Nesvorný, D., \& Bottke, W. F. 2003, Nature, 425, 147

Vokrouhlický, D., Breiter, S., Nesvorný, D., \& Bottke, W. F. 2007, Icarus, in press 